\title{
A Case of Solitary Pulmonary Nodule that Presented with Chronic Cough: What Is Your Diagnosis?
}

\author{
Banu M. Salepci
}

Department of Pulmonary Diseases, Dr. Lütfi Kırdar Kartal Education and Research Hospital, İstanbul

A sixty year old female patient was admitted to our clinic with a complaint of chronic non-productive cough for 3 months. The patient, who was a physician herself, had a 40 pack.year history of smoking, and her medical history was unremarkable except for short term use of antibiotics and expectorants, following the start of her cough complaint. She had no other disease. In the physical examination, thorax was symmetrical and both hemithoraces contributed equally to respiration. Auscultation revealed rare bilateral rhonchi and mildly prolonged expirium. Vital findings, oxygen saturation and electrocardiogram readings of the patient were in normal range, and PPD test was $17 \mathrm{~mm}$. In pulmonary function testing, there were no findings other than mild obstructive dysfunction.

Posteroanterior chest X-ray showed bilateral hyperaeration and a 15 $\mathrm{mm}$ solitary pulmonary nodule with slightly irregular margins at the

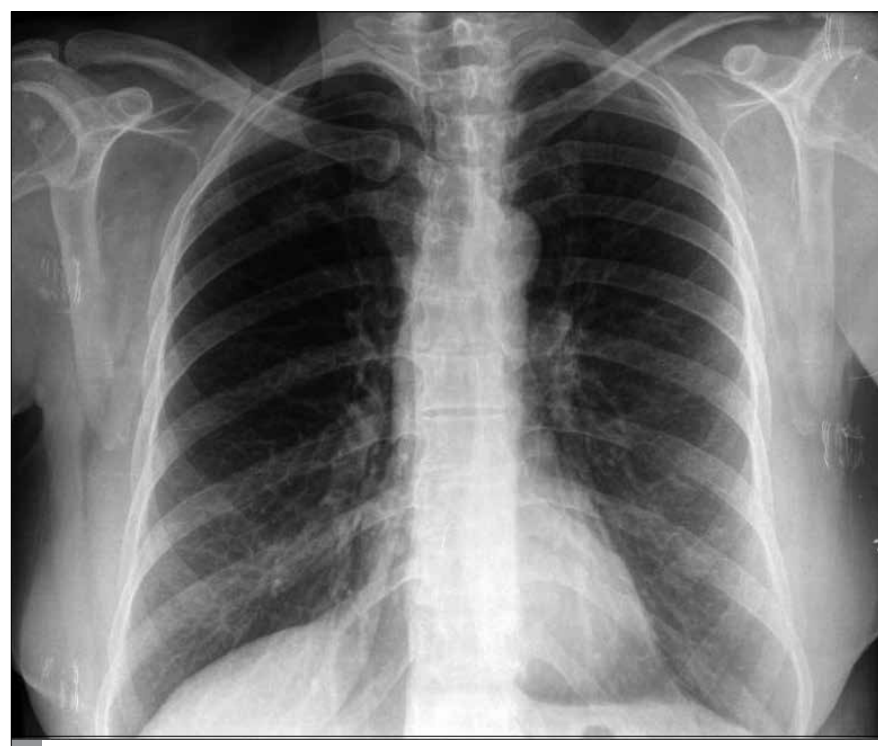

Figure 1. The postero-anterior chest X-ray of the patient showing a round nodule with indistinct margins, at the right upper zone right upper lobe (Figure 1). The patient having risk factors including cigarette smoking and airway obstruction was scheduled for computed tomography of the thorax (thorax CT) with a pre-diagnosis of malignancy. In the thorax CT, a solid nodule with spiculated margins was detected at the posterior segment of the right upper lobe at the parenchymal window. There was no other radiological finding such as parenchymal infiltration, atelectasis and pleural invasion. Mediastinal window images of the thorax CT revealed a nodule with soft tissue density and lobulated contours (Figure 2 and 3).

No abnormality was detected in the video bronchoscopic investigation of the patient and bronchoalveolar lavage fluid obtained from the right upper lobe revealed no malignant cells. In the positron emission tomography (PET), the SUVmax of the $15 \mathrm{~mm}$ nodule was reported to be 5.5 , and there were no hypermetabolic lymph nodes or any

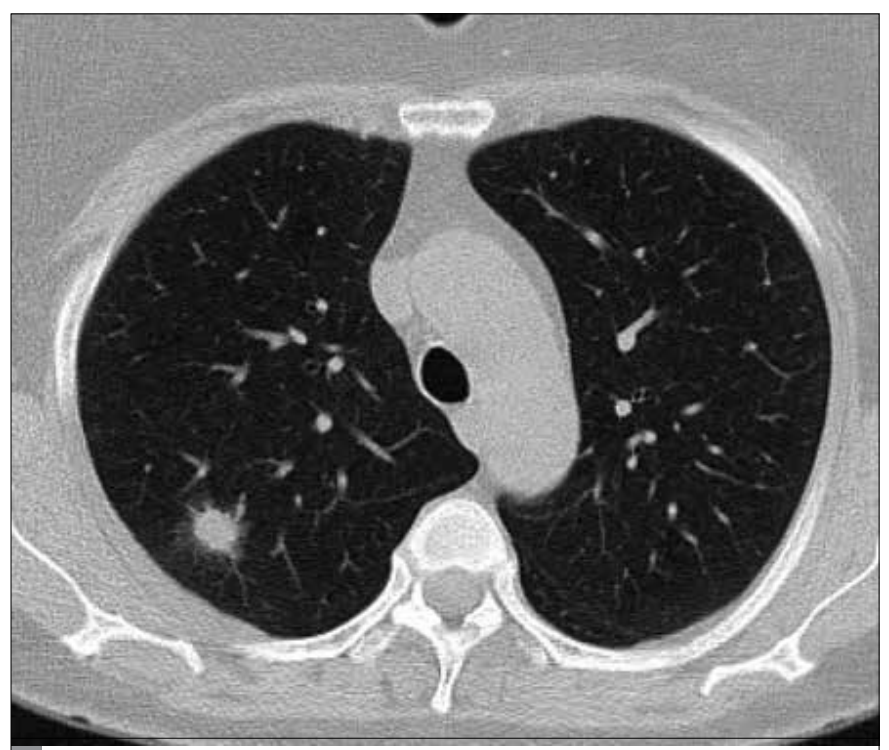

Figure 2. Computed tomography of the thorax (parenchymal window) showing a nodule with spiculated margins localized at the posterior segment of the right upper lobe 


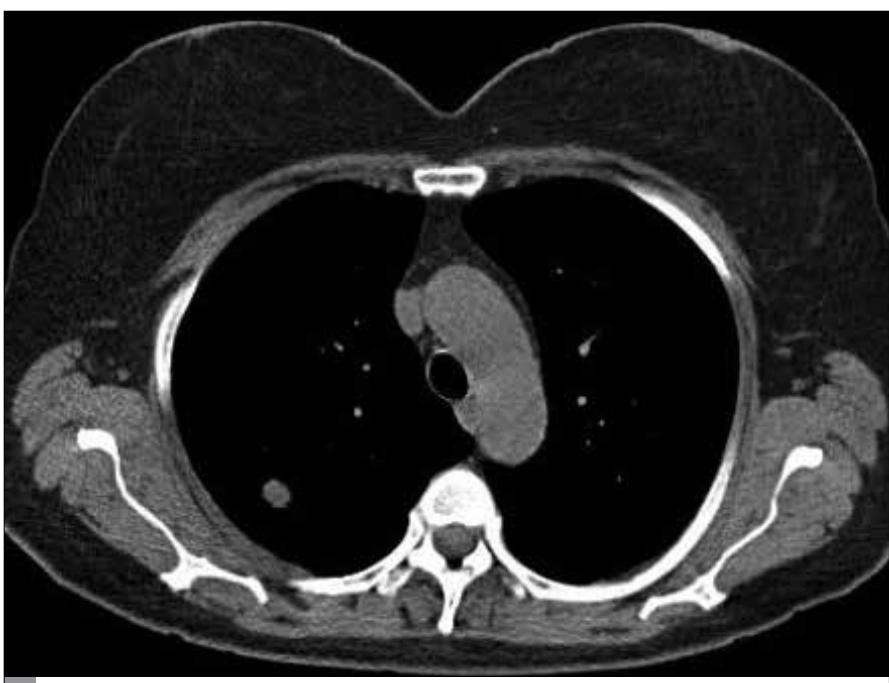

Figure 3. Computed tomography of the thorax (mediastinal window) showing a solid lobulated nodule

other foci. According to these findings, diagnostic intervention, CT-guided transthoracic fine-needle aspiration biopsy (FNAB) was performed. The cytological evaluation of the biopsy material showed inflammatory cells. As the FNAB results of the patient with a high probability of malignancy were not diagnostic, the nodule was removed by wedge resec-

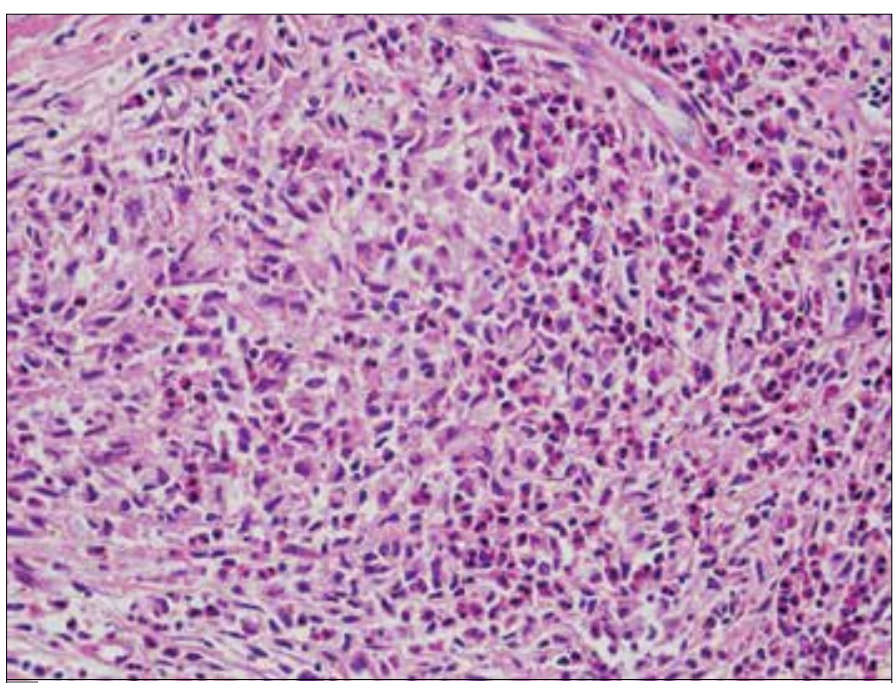

Figure 4. (H\&Ex400): Pale, granular cells with indistinct cytoplasmic margins and basophilic nuclei (arrow) and eosinophilic infiltration

tion. Since the nodule was reported as non-malignant in the intraoperative frozen section investigation, the surgical operation was completed without performing a lobectomy. Post-operative pathological investigation of the nodule showed eosinophilic infiltration and pale, granular, basophilic nuclei cells with indistinct cytoplasmic margins (Figure 4). 


\section{What is your diagnosis for this patient?}

A solitary pulmonary nodule (SPN) is defined as a single, well-marginated, round radiological opacity not bigger than $3 \mathrm{~cm}$ in diameter, that is surrounded by lung parenchyma, and is not associated with hilar adenopathy, atelectasis, or pleural effusion (1). The probability of malignancy of solitary pulmonary nodules is approximately between $40 \%$ and $50 \%$ above 40 years of age. Variables like age of the patient, history of cigarette smoking, dimensions and characteristics of the nodule form the basis for diagnostic strategy and the nodule can either be followed up radiologically or can be resected by surgery and diagnosed histopathologically (2). As because the patient was above the age of 40 years, had a history of cigarette smoking, a nodule with no benign calcification patterns demonstrating spiculated margins at thorax CT and hypermetabolism (SUV max: 5.5) at PET/CT, she had a high probability of malignancy. Hence, rather than choosing the "follow-up and see" strategy, we preferred to remove the nodule with surgical operation. The histopathological investigation of the nodule, $1.5 \times 1.5 \times 1.0 \mathrm{~cm}$ in size, revealed eosinophilic infiltration, granulomas composed of eosinophils and, pale, granular Langerhans cells with indistinct cytoplasmic margins, and basophilic nuclei. In the immunohistochemical analysis, cells were positive for S100 and CD1a, weakly positive for CD68 and negative for CK. On account of these findings, our case was diagnosed as having pulmonary eosinophilic granuloma (PEG) (pulmonary Langerhans cell histiocytosis). Terminologically, histiocytosis refers to proliferative diseases of histiocytes. At one end of the spectrum are aggressive malignant diseases such as malignant histiocytic lymphoma, and at the other end histiocytic proliferation in lymph nodes. Between these two end-points, is the disease called eosinophilic granuloma or Langerhans Cell Histiocytosis that can be defined as the proliferation and infiltration of a special histiocyte group called Langerhans cells (3). This disease, which has an asymptomatic course or present with mild complaints, is mostly seen in smokers (4).

In pulmonary eosinophilic granuloma, the usual radiological pattern is bilateral, diffuse and symmetrical opacities that are predominant in the upper lobe. These opacities may be cystic, nodular, reticulonodular or reticular. Apart from that, cases with pleural effusion and hilar enlargement have also been reported (2).

On rare occasions, PEG may present as a solitary pulmonary nodule; these cases have been associated with cigarette smoking and they have no specific symptoms at the time of diagnosis (3).

As our patient had no pulmonary radiological findings other than the nodule in the right upper lobe and as no other organ involvement was detected, additional treatment was not considered and the case was decided to be followed up.

Address for correspondence: Banu M. Salepci, Department of Pulmonary Diseases, Dr. Lütfi Kırdar Kartal Education and Research Hospital, İstanbul, Turkey E-mail: bsalepci@yahoo.com

๑) Copyright 2014 Turkish Respiratory Society (TRS)

DOI: 10.5152/ejp.2014.09581

- Available online at www.eurasianjpulmonol.com

\section{REFERENCES}

1. Ost $D E$, Gould MK. Decision making in patients with pulmonary nodules. Am J Resp Crit Care Med 2012; 185: 363-72. [CrossRef]

2. Velde, G.P.M.ten, Thunnissen F.B.J.M., Engelshoven van J.M.A., Wouters E.F.M. A solitary pulmonary nodule due to eosinophilic granuloma. Eur Respir J 1994; 7: 1539-40. [CrossRef]

3. Vassallo Robert, Andrew H.Limper. Pulmonary langerhans cell histiocytosis. In: Schwartz Marvin I, King TE, editors. Interstitial lung disease. 4th ed. London: BC Decker Inc; 2003.p.838-50.

4. Fichtenbaum CJ, Kleinman GM, Haddad RG. Eosinophilic granuloma of the lung presenting as a solitary pulmonary nodule. Thorax 1990; 45: 905-6. [CrossRef] 